\title{
Robot Flocking with Communication Noise
}

\author{
LIU Bin \\ School of Mechanical Engineering, Nanjing Institute of \\ Industry Technology, Nanjing Jiangsu 210046, China \\ liub@niit.edu.cn
}

\author{
PENG Huan-xin \\ School of Mechanical Engineering, Nanjing Institute of \\ Industry Technology, Nanjing Jiangsu 210046, China \\ penghx@niit.edu.cn
}

\begin{abstract}
In the paper, we analyze the robot flocking algorithms under virtual leader-follower model with communication noise. Under virtual leader-follower model, robot flocking algorithm can avoid diverging, but the performance of robot flocking algorithm with communication noise often degrades. In the paper, we analyze the impact brought by communication noise on the robot flocking, and simulations are done. Results show that the performance of robot flocking depends on the communication noise. Smaller is the communication noise, better is the performance of robot flocking with communication noise.
\end{abstract}

Keywords-robot; flocking; communication noise; virtual leader-follower

\section{INTRODUCTION}

In nature, based on local information, birds, bees, and fish often flock together in groups to find the source of food or avoid predators. This natural phenomenon of flocking has been studied in the past years [1-7]. Reynolds proposed the Boid model in [1] about the phenomenon of flocking.

The problems of flocking have also attracted many researchers in many fields. Viscek et al are the first groups of physicists who studied flocking from a theoretical perspective. Viscek in [2] propose a model based on statistical mechanics, the model only considers flock centering rule of Boid model. Jadbabaie simplify the Viscek model by linear approximation in [3]. In [4], the theoretical framework for design and analysis of distributed flocking algorithms was proposed.

For the limited communication radius and other reasons, the flocking algorithm is easy to diverge. In order to solve the problem, Olafi-saber in [4] proposes leader-follower model to void diverging. In the leader-follower model, every agent can receive the information of the virtual leader. As an extension of the flocking algorithm in [4], flocking of agents with a virtual leader in case of a minority of informed agents and varying velocity of virtual leader was presented in [7].

Recently, the flocking algorithm [1-7] is applied in robot field, robots communicate with each other, and every robot only can receive the information from the nearby robots. For the limited communication radius, not all robots can receive the information from the nearby robots, so the robots are easy to diverge. In order to solve the problem, the robots flocking based on leader-follower model is supposed. Under the leaderfollower model, the performance of the robots flocking improves.

For robots flocking, in the course of utilizing the information of the nearby robots, there is often communication noise, robots flocking with communication noise is easy to diverge, meantime, there is noise for every robot to receive the information of virtual leader, and the impact of noise on robots flocking has to be considered.

In the paper, we consider robots flocking with communication noise among agents under leader-follower model. The communication noise is supposed to be zero-mean Gaussian white noise, and irrelevant and independent in time and spatial, meantime, we suppose that all robots can receive the information of virtual leader. The performance of robots flocking with noises is analyzed, and simulations are done.

The remainder of this paper is organized as follows: we introduce some necessary notations about graph theory and the robots flocking. We explicitly analyze the performance of the robots flocking with communication noise. Examples and simulation results are provided and conclusions are summarized in the end.

\section{FLOCKING ALGORITHM UNDER VIRTUAL LEADER}

Firstly, we introduce some notations and concepts about distributed consensus and distributed flocking algorithm. A graph $G=(V, E)$ represents the communication topology in a networked multi-agent system with $n$ agents, where $V$ is a set of vertices, and $E$ is a set of edges. Each edge in the graph is denoted by $(i, j) \in E$, where $i, j \in V$. An adjacency matrix for graph $G$ is denoted as $A=\left\{a_{i j}\right\}_{N \times N}$, where $a_{i j}=\left\{\begin{array}{ll}1 & (i, j) \in E \\ 0 & (i, j) \notin E\end{array}\right\}$. A set of vertices $N_{i}$ is denoted as the set of vertices that can send directly information to vertex $i$. A Laplacian matrix is denoted as $L=\left\{l_{i j}\right\}=\Delta-A$, where $\Delta=\operatorname{diag}\left\{\sum_{j=1}^{N} a_{1 j} \ldots . . \sum_{j=1}^{N} a_{N j}\right\}$.

A continuous-time first-order consensus algorithm in [8] can be represented by:

$$
\dot{x}_{i}(t)=-\sum_{j \in N_{i}} a_{i j}\left(x_{i}(t)-x_{j}(t)\right)
$$

Suppose by Precision manufacturing engineering technology research center foundation of Nanjing Institute of Industry Technology (ZK13-01-01) 
The collective dynamics of the group of agents can be written as:

$$
\dot{x}(t)=-L x(t)
$$

where $x(t)=\left[x_{1}(t), x_{2}(t) \ldots, x_{n}(t)\right]^{T}$, and $x_{i}(t)$ is denoted by the state of agent $i$

Based on Boid model's three rules, we consider the velocity and position of agents, the dynamics of the agent $i$ can be written by:

$$
\dot{q}_{i}=p_{i}, \quad \dot{p}_{i}=u_{i}
$$

Where $q_{i} \in R^{m}$ is denoted by the position of agent $i$, and $p_{i} \in R^{m}$ is denoted by the velocity of agent $i$, and $u_{i} \in R^{m}$ is denoted by the control input of agent $i$.When agent move, the communication topology changes, we define the communication topology as $G(t)$ in time $t$, and the corresponding Laplacian matrix is $L(t)$, obviously, for the undirected topology, $L(t)$ is a positive semi-definite matrix .when graph $G(t)$ is undirected and connected, then the second Minimum eigen-value of matrix $L(t)$, i.e., $\lambda_{2}(L(t))>0$.

For leader-follower model, considering agent $i$, the control input is:

$$
u_{i}=f_{i}^{g}+f_{i}^{d}+f_{i}^{\gamma}
$$

where $f_{i}^{g}$ is denoted by a gradient term, and $f_{i}^{g}=-\nabla_{q_{i}} V(q)$, where $V(q)$ is the collective potential function. The item $f_{i}^{d}$ is a velocity consensus/alignment term that acts as a damping force, and $f_{i}^{\gamma}$ is a navigational feedback due to a group objective. Examples of a group objective are moving towards a destination (migration) or flocking out of a crowded space with few exits. The collective potential function $V(q)$ of a group of agents is a non-negative function $V(q): R^{m n} \rightarrow R$ with the property that any solution of the set of algebraic constraints in (7) is "closely related to" a local minimum of $V(q)$ and vice versa [4]. When the distance between two agents: $\left\|q_{i}-q_{j}\right\| \rightarrow 0$, then $V\left(q_{i}\right)$ is maximum, when the distance $\left\|q_{i}-q_{j}\right\|$ reach a expectation value, i.e., the distance between two agents reach a expectation value, then $V\left(q_{i}\right)$ is minimum, when the distance is greater than the expectation value, then $V\left(q_{i}\right)$ retain a very small positive number.

For the virtual leaders, the dynamics can be written by:

$$
\left\{\begin{array}{c}
\dot{q}_{r}=p_{r} \\
\dot{p}_{r}=f_{r}\left(q_{r}, p_{r}\right)
\end{array}\right.
$$

where the pair $\left(q_{r}, p_{r}\right) \in R^{m} \times R^{m}$ is the state of virtual leader. The virtual leader is dynamic/static agent that represents a group objective. The initial state of virtual leader is denoted by $\left(q_{r}(0), p_{r}(0)\right)=\left(q_{d}, p_{d}\right)$. A static virtual leader has a fixed state that is equal to $\left(q_{d}, p_{d}\right)$ for all time.

For undirected graph $G(V, E)$, we define the communication radius among agents as $r$, and we know that during the movement of agents, when the relative distance between agents changes, hence the neighbors of each agent also change. Therefore, we can define a neighborhood set of agent $i$ as follows:

$$
N_{i}=\left\{j\left\|\mid q_{i}-q_{j}\right\|_{2}<r\right\}
$$

where $\|\cdot\|_{2}$ is the Euclidean norm, the geometry of flocks is modeled by $\alpha$-lattice [4] that meets the following condition:

$$
\left\|p_{i}-p_{j}\right\|_{2}=d, \quad j \in N_{i}
$$

where $d$ is a positive constant indicating the distance between agent $i$ and its neighbor $j$

The $\sigma-$ norm of a vector is a map $R^{m} \rightarrow R$ defined as:

$$
\|z\|_{\sigma}=\frac{1}{\kappa}\left(\sqrt{1+\kappa\|z\|_{2}^{2}}-1\right)
$$

Where the parameter $\kappa>0$, the gradient $\sigma_{\varepsilon}(z)=\nabla\|z\|_{\sigma}$ is defined as:

$$
\sigma_{\kappa}(z)=\frac{z}{\sqrt{1+\kappa\|z\|_{2}^{2}}}=\frac{z}{1+\kappa\|z\|_{\sigma}}
$$

The $\sigma-$ norm $\|z\|_{\sigma}$ is differentiable everywhere. This property allows to construct a smooth collective potential function for agents. The $\sigma_{\varepsilon}(z)$ is a uniformly bounded function satisfying $z^{T} \sigma_{\varepsilon}(z)>0, \forall z \neq 0,\left\|\sigma_{\varepsilon}(z)\right\|_{2}<1 / \sqrt{\varepsilon}$, and $(x-y)^{T}\left(\sigma_{1}(x)-\sigma_{1}(y)\right)>0, \forall x \neq y$.

Pump function $\rho_{h}(z)$ varies smoothly between 0 and 1 .

$$
\rho_{h}(z)=\left\{\begin{array}{cc}
1 & z \in[0, h) \\
\frac{1}{2}\left(1+\cos \left(\pi \frac{(z-h)}{(1-h)}\right)\right) & z \in[h, 1] \\
0 & \text { otherwise }
\end{array}\right.
$$


where $h \in(0,1)$. Based on the pump function, the spatial adjacency matrix $A(q)$ can be defined via its elements by:

$$
a_{i j}(q)=\rho_{h}\left(\left\|q_{j}-q_{i}\right\|_{\sigma} / r_{a}\right) \in[0,1], \quad i \neq j
$$

where $r_{a}=\|r\|_{\sigma}, a_{i i}(q)=0$. If $h=1$, the pump function $\rho_{h}(z)$ is a indictor function. The collective potential function $V(q)$ can be written by:

$$
\begin{aligned}
& V(q)=\frac{1}{2} \sum_{i} \sum_{j \neq i} \psi_{\alpha}\left(\left\|q_{j}-q_{i}\right\|_{\sigma}\right) \\
& \psi_{\alpha}(z)=\int_{d_{\alpha}}^{z} \varphi_{\alpha}(s) d s \\
& \varphi_{\alpha}(z)=\rho_{h}\left(z / r_{\alpha}\right) \varphi\left(z-d_{\alpha}\right), \\
& \varphi(z)=\frac{1}{2}\left[(a+b) \sigma_{1}(z+c)+(a-b)\right]
\end{aligned}
$$

where $d_{\alpha}=\|d\|_{\sigma}, r_{\sigma}=\|r\|_{\sigma}, \sigma_{1}(z)=z / \sqrt{1+z^{2}}$, and $0<a \leq b, c=|a-b| / \sqrt{4 a b}$.

\section{ROBOTS FLOCKING WITH COMMUNICATION NOISE UNDER VIRTUAL LEADER-FOLLOWER}

In the paper, we analyze robot flocking problem with communication noise based on the virtual leader-follower model. There is noise among robots and among leaders and followers. We suppose the noise among robots is zero-mean Gaussian white noise, and the noise is irrelevant and independent in time and spatial, meantime, we suppose that all robots can receive the information of virtual leader.

The robots flocking with noise based on leader-follower model can be written by:

$$
\begin{aligned}
u_{i}(k) & =f_{i}^{g}(k)+f_{i}^{d}(k)+f_{i}^{\gamma}(k) \\
f_{i}^{d}(k) & =-\sum_{j \in N_{i}} a_{i j}(q(k))\left\{\left(p_{i}(k)-p_{j}(k)+v_{j}(k)\right)\right. \\
f_{i}^{g}(k) & =\sum_{j \in N_{i}} \varphi_{\alpha}\left(\left\|q_{j}(k)-q_{i}(k)+w_{j}(k)\right\|_{\sigma}\right) \\
& \times \frac{q_{j}(k)-q_{i}(k)+w_{j}(k)}{\sqrt{1+\kappa\left\|q_{j}(k)-q_{i}(k)+w_{j}(k)\right\|_{2}^{2}}} \\
\varphi_{\alpha}(z) & =\rho_{h}\left(z / r_{\alpha}\right) \varphi\left(z-d_{\alpha}\right), \\
\varphi(z) & =\frac{1}{2}\left[(a+b) \sigma_{1}(z+c)+(a-b)\right]
\end{aligned}
$$

$\left\|q_{j}(k)-q_{i}(k)\right\|_{\sigma}=d_{\alpha}=\|d\|_{\sigma}=\frac{1}{\kappa}\left[\sqrt{1+\kappa\|d\|_{2}^{2}}-1\right]$

$\sigma_{1}(z)=z / \sqrt{1+z^{2}}$

Where $w_{j}(k), v_{j}(k)$ is zero-mean Gaussian white noise, and the noise is irrelevant and independent in time and spatial, i.e.:

$$
\begin{aligned}
& E\left(v_{j}(k)\right)=0, \quad E\left(v_{i}(k) v_{j}(k)\right)=\left\{\begin{array}{cc}
0 & i \neq j \\
\sigma_{v i}^{2}(k) & i=j
\end{array}\right\}, \\
& E\left(v_{i}(k) v_{i}(m)\right)=\left\{\begin{array}{cc}
0 & m \neq k \\
\sigma_{v i}^{2}(k) & m=k
\end{array}\right\}, E\left(w_{j}(k)\right)=0, \\
& E\left(w_{i}(k) w_{j}(k)\right)=\left\{\begin{array}{cc}
0 & i \neq j \\
\sigma_{w i}^{2}(k) & i=j
\end{array}\right\}, \\
& E\left(w_{i}(k) w_{i}(m)\right)=\left\{\begin{array}{cc}
0 & m \neq k \\
\sigma_{w i}^{2}(k) & m=k
\end{array}\right\} .
\end{aligned}
$$

For the virtual leaders, the dynamics can be written by:

$$
\left\{\begin{array}{c}
\dot{q}_{r}=p_{r} \\
\dot{p}_{r}=f_{r}\left(q_{r}, p_{r}\right)
\end{array}\right.
$$

where the pair $\left(q_{r}, p_{r}\right) \in R^{m} \times R^{m}$ is the state of virtual leader. The virtual leader is dynamic/static agent that represents a group objective. The initial state of virtual leader is denoted by $\left(q_{r}(0), p_{r}(0)\right)=\left(q_{d}, p_{d}\right)$. A static virtual leader has a fixed state that is equal to $\left(q_{d}, p_{d}\right)$ for all time.

The collective form can be written by:

$\left\{\begin{array}{c}q(k+1)=q(k)+\varepsilon p(k) \\ p(k+1)=p(k)-\varepsilon \tilde{L}(k)(p(k)+v(k)-\varepsilon \hat{L}(q(k))(q(k)+w(k))\end{array}\right.$

Where $w(k), v(k)$ is a vector, $\tilde{L}(k)=L(k) \otimes \mathbf{1}^{m}$, the corresponding adjacency matrix $A=\left\{a_{i j}(q(k))\right\}_{n \times n}$, and $\hat{L}(q(k))=L(q(k)) \otimes \mathbf{1}^{m}$, the corresponding adjacency matrix $A(q(k))=\left\{\beta_{i j}(k)\right\}_{n \times n}$, and the parameter is equal to:

$$
\begin{array}{r}
\beta_{i j}(k)=\frac{\varphi_{\alpha}\left(\left\|q_{j}(k)-q_{i}(k)+w_{j}(k)\right\|\right)}{\sqrt{1+\varepsilon\left\|q_{j}(k)-q_{i}(k)+w_{j}(k)\right\|_{2}^{2}}} . \\
\text { IV. SIMULATIONS AND ANALYSES }
\end{array}
$$

We present simulation results to illustrate the performance of our proposed the robot flocking with communication noise based on virtual leader-follower model. For simplicity's sake, we only consider 2-D flocking for 100 robots in the free space. 
The initial position of robots is stay between $-50(\mathrm{~m})$ and $50(\mathrm{~m})$ in the $x$ axis, and $-50(\mathrm{~m})$ and $50(\mathrm{~m})$ in the $y$ axis. The initial velocity of agents is stay between $-5(\mathrm{~m} / \mathrm{s})$ and $5(\mathrm{~m} / \mathrm{s})$ in the $\mathrm{x}$ axis, and $-5(\mathrm{~m} / \mathrm{s})$ and $5(\mathrm{~m} / \mathrm{s})$ in the $y$ axis. The parameters $a=b=4, \kappa=0.2$, and sampling period $\varepsilon=0.02$ (s), $h=0.1, c_{1}=c_{2}=0.5$. The communication radius among agents is $25 \mathrm{~m}$, and the expectation distance between robots is: $d=20$.

The three graph show that the velocity of robots on $\mathrm{x}$ axis and the velocity of robots on $y$ axis under different meansquare error.
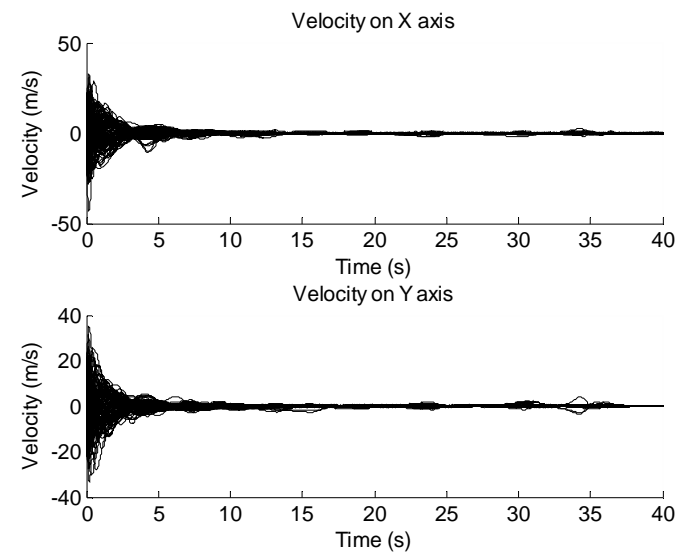

Fig.1. Velocity of robots with the $E\left(v_{i}(k) v_{i}(k)\right)=1$ and $E\left(w_{i}(k) w_{i}(k)\right)=1$

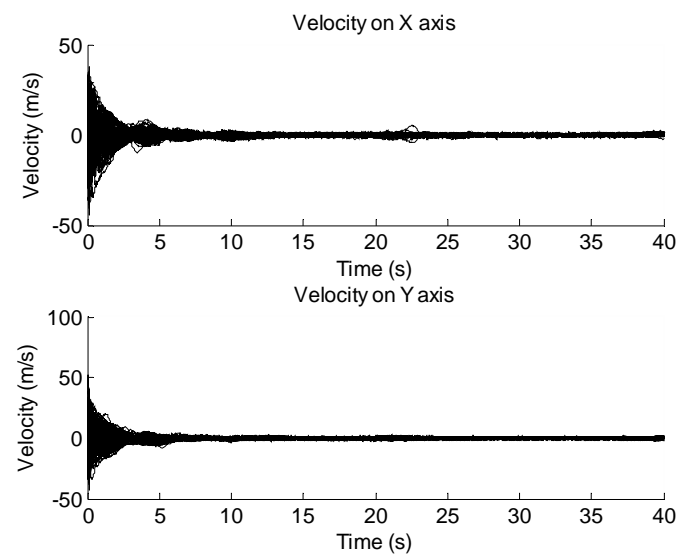

Fig.2. Velocity of robots with the $E\left(v_{i}(k) v_{i}(k)\right)=10$ and $E\left(w_{i}(k) w_{i}(k)\right)=10$

Fig. 1 shows velocity of robots on the $\mathrm{x}$ axis and $\mathrm{y}$ axis when the mean square value of noise is $E\left(v_{i}(k) v_{i}(k)\right)=1$, and $E\left(w_{i}(k) w_{i}(k)\right)=1$. Fig. 2 shows velocity of robots on the $\mathrm{x}$ axis and $y$ axis when the mean square value of noise is $E\left(v_{i}(k) v_{i}(k)\right)=10$, and $E\left(w_{i}(k) w_{i}(k)\right)=10$. Fig. 3 shows velocity of robots on the $\mathrm{x}$ axis and $\mathrm{y}$ axis when the mean square value of noise is $E\left(v_{i}(k) v_{i}(k)\right)=100$, and $E\left(w_{i}(k) w_{i}(k)\right)=100$.
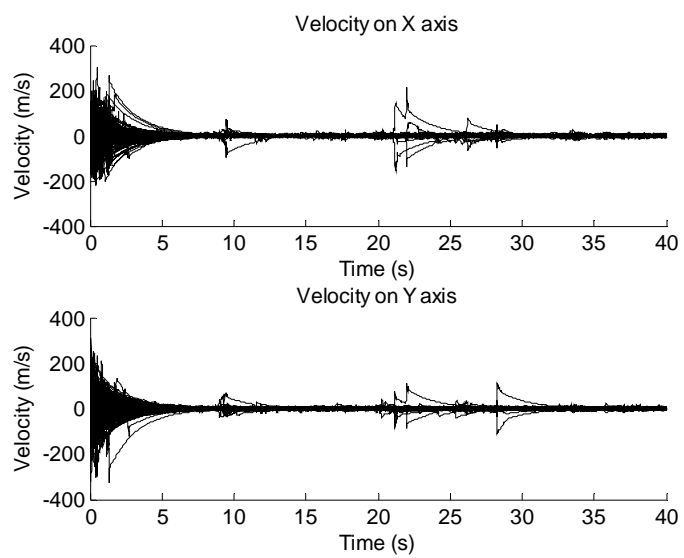

Fig.3. Velocity of robots with the $E(v(k) v(k))=100$ and $E(w(k) w(k))=100$

Obviously, the robots can converge under communication noise, but bigger the mean square error of the communication noise is, worse the performance of robots flocking is. Under the leader-follower model, every robot can receive the information of the virtual leader, although there is communication noise among robots, the velocity of all robots can receive cohesion.

\section{CONCLUSIONS}

In the paper, we analyze the performance of robots flocking with communication noises. Through simulations, the results show all robots with communication noise can receive cohesion under leader-follower model, and bigger are the noises, then, poorer is the performance of robots flocking.

\section{REFERENCES}

[1] C. W. Reynolds. "Flocks, herds, and schools: a distributed behavioral model,” Computer Graphics, vol. 21, no. 4, pp. 25-34, 1987.

[2] T. Vicsek, A. Cziro'ok, E. Ben-Jacob,et.al. "Novel type of phase transition in a system of self-deriven particles," Physical Review Letters, vol. 75, no. 6, pp. 1226-1229, 1995.

[3] A. Jadbabaie, J. Lin, A. S. Morse. "Coordination of groups of mobile autonomous agents using nearest neighbor rules," IEEE Transactions on Automatic Control, vol. 48, no. 6, pp. 988-1001, 2003.

[4] R. Olfati-Saber. "Flocking for multi-agent dynamic systems: Algorithms and theory,” IEEE Transactions on Automatic Control, vol. 51, no. 3, pp. 401-420, 2004.

[5] H. G. Tanner, A. Jadbabaie, G. J. Pappas. "Stable flocking of mobile agents, Part I: Fixed topology,” In Proceedings of the IEEE Conference on Decision and Control, Maui, USA: IEEE, 2003, pp. 2010-2015.

[6] H. G. Tanner, A. Jadbabaie, G. J. Pappas. "Flocking in fixed and switching networks,” IEEE Transactions on Automatic Control, vol. 52, no. 5, pp.863-868, 2007.

[7] H. S. Su, X. F. Wang, Z. L. Lin. "Flocking of multi-agents with a virtual leader,” IEEE Transaction Automatic Control, vol. 54, no. 2, pp. 293307, 2009.

[8] R. Olfati-Saber, R. M. Murray, "Consensus problems in networks of agents with switching topology and noises," IEEE Transaction on Automatic Control, vol. 49, no. 9, pp.1520-1533, 2004. 